\title{
Small RNA molecules in the regulation of spermatogenesis
}

\author{
Zuping He, Maria Kokkinaki, Disha Pant, G Ian Gallicano and Martin Dym \\ Department of Biochemistry and Molecular and Cellular Biology, Georgetown University Medical Center, \\ 3900 Reservoir Road NW, Washington, District of Columbia 20057, USA
}

Correspondence should be addressed to M Dym; Email: dymm@georgetown.edu

\begin{abstract}
Small RNA molecules (small RNAs), including small interfering RNAs (siRNAs), microRNAs (miRNAs), and piwi-interacting RNAs (piRNAs), have recently emerged as important regulators of gene expression at the post-transcriptional or translation level. Significant progress has recently been made utilizing small RNAs in elucidating the molecular mechanisms regulating spermatogenesis. Spermatogenesis is a complex process that involves the division and eventual differentiation of spermatogonial stem cells into mature spermatozoa. The process of spermatogenesis is composed of several phases: mitotic proliferation of spermatogonia to produce spermatocytes; two meiotic divisions of spermatocytes to generate haploid round spermatids; and spermiogenesis, the final phase that involves the maturation of early-round spermatids into elongated mature spermatids. A number of miRNAs are expressed abundantly in male germ cells throughout spermatogenesis, while piRNAs are only present in pachytene spermatocytes and round spermatids. In this review, we first address the synthesis, mechanisms of action, and functions of siRNA, miRNA, and piRNA, and then we focus on the recent advancements in defining the small RNAs in the regulation of spermatogenesis. Concerns pertaining to the use of siRNAs in exploring spermatogenesis mechanisms and open questions in miRNAs and piRNAs in this field are highlighted. The potential applications of small RNAs to male contraception and treatment for male infertility and testicular cancer are also discussed.
\end{abstract}

Reproduction (2009) 137 901-911

\section{Introduction}

Mammalian spermatogenesis begins with the selfrenewal and differentiation of spermatogonial stem cells (SSCs). In the rodent testis as illustrated in Fig. 1, SSCs can divide into either new stem cells $\left(A_{s}\right.$ spermatogonia) or type $A_{\text {paired }}\left(A_{p r}\right)$ spermatogonia that then produce the type $A_{\text {aligned }}\left(A_{a l}\right)$ spermatogonia (de Rooij \& Grootegoed 1998). The $A_{a l}$ spermatogonia, in turn, give rise to several generations of spermatogonia, including type $\mathrm{A}_{1}-\mathrm{A}_{4}$, intermediate, and type $B$ spermatogonia (Fig. 1). Type $B$ cells form primary spermatocytes followed by secondary spermatocytes, which eventually generate early and late spermatids (Fig. 2; Bellvé et al. 1977).

Small RNA molecules (small RNAs) are important because of their diverse biological functions as potent regulators of transcription, RNA stability, and translation (Plasterk 2006). An attractive new area for investigating the mechanisms by which spermatogenesis is regulated involves small RNAs that suppress gene expression, and a number of endogenous small RNAs have been identified in male germ cells throughout spermatogenesis. Classification of small RNAs (Table 1 ) is based upon their biogenesis, mechanisms of action, and functions (Tolia \& Joshua-Tor 2007), and there are three major small RNAs: small interfering RNA (siRNA); microRNA (miRNA); and piwi-interacting RNA (piRNA). Recent studies in various eukaryotes have shown that indeed these small RNAs play major roles in the control of gene expression.

The siRNA is a small ( $\sim 21$ nucleotides (nt) in length) double-stranded RNA (dsRNA), and a short hairpin RNA (shRNA) is a sequence of RNA that makes a tight hairpin turn and it can silence the expression of genes. RNA interference (RNAi) using siRNAs or shRNA has been demonstrated as a useful approach to effectively knock down the expression of a particular gene and analyze the effect that gene has on cellular function. siRNA has been used as an alternative to knocking out genes in mice. While the gene knockout approach is useful for clarifying the physiological roles of individual genes, it is laborious and costly compared to the gene knockdown approach using RNAi. In addition, it is rather hard to study the functions of a number of genes since gene knockout often results in lethal embryos or the death of newborn pups.

miRNAs were identified as a different class of small ( $\sim 22 \mathrm{nt}$ in length) RNA molecules. Although the miRNAs first discovered in 1993 in Caenorhabditis elegans were found to regulate the expression of 


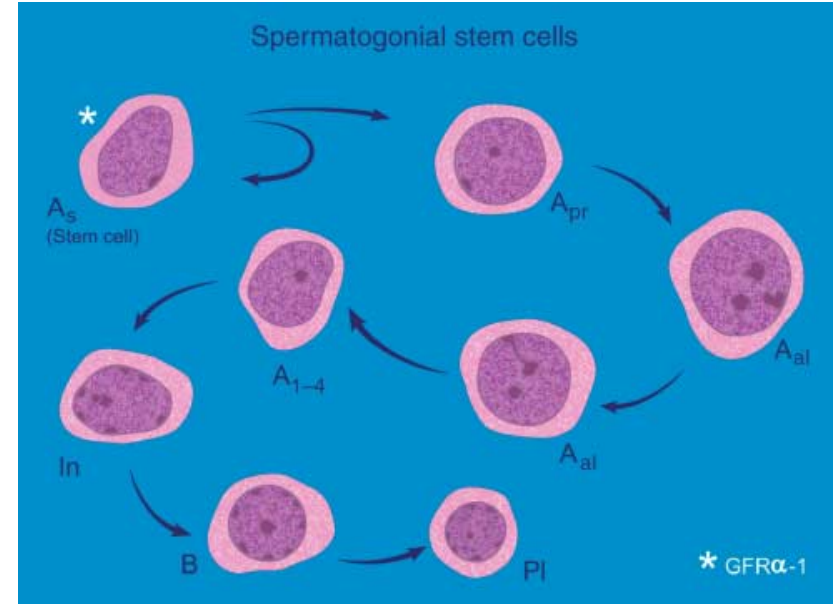

Figure $1 \mathrm{~A}$ proposed scheme of stem cell renewal in rodent testis. The $A_{s}$ spermatogonia are considered as the stem cells in the testis, while the $A_{p r}$ and $A_{a l}$ spermatogonia are the progenitor cells. In this scheme, the $A_{s}, A_{p r}$ and $A_{a l}$ are also considered morphologically undifferentiated spermatogonia, whereas type $A_{1-4}$ spermatogonia, intermediate (In) spermatogonia, and type B spermatogonia are differentiating spermatogonia. Modified from Huckins (1971) and Oakberg (1971).

complementary mRNA (Lee et al. 1993, Wightman et al. 1993), it was only 8 years ago that miRNAs were identified in mammals (Lagos-Quintana et al. 2001). Hundreds of miRNAs (672 in human and 599 in mice miRBase Release 11.0, as of April, 2008, http://microrna. sanger.ac.uk/sequences/) have been discovered, and it is likely that $\sim 1000$ miRNAs are present in each of the human and mouse genomes. miRNAs are highly conserved across species, and importantly, it has been estimated that miRNAs may regulate up to $30 \%$ of all genes in the human genome (Lewis et al. 2005).
Another newly identified class of small RNAs is called piRNAs because these small RNAs interact with piwifamily proteins, such as MIWI, MIWI2, and MILI (Aravin et al. 2006, Girard et al. 2006, Grivna et al. 2006a, 2006b). Piwi-family proteins refer to a group or family of proteins that have amino acid sequences, which are substantially identical to the native amino acid sequences in the piwi family, and they include PIWI, HIWI, MIWI, MIWI2, MILI, PRG-1, and PRG-2 proteins. Distinct from the siRNAs or miRNAs, piRNAs are 24-30 nt in length and they are present in pachytene spermatocytes and spermatids during spermatogenesis, and are required for germline development in both males and females (Klattenhoff \& Theurkauf 2008). Currently, there are $5 \times 10^{4}$ piRNAs that have already been discovered, and it is estimated that the total number of piRNAs is around $2 \times 10^{5}$ (Betel et al. 2007), suggesting that piRNAs may be essential for a broad range of biological processes (Klattenhoff \& Theurkauf 2008). Notably, a piRNA Bank containing the known piRNAs of human, mouse, and rat is now available from the website: http://pirnabank.ibab.ac.in.

Small RNAs (siRNAs, miRNAs, and piRNAs) have recently been used in elucidating the molecular mechanisms regulating spermatogenesis, in particular endogenous genes in germ cells that regulate the complex process of their renewal and/or differentiation. In this review, we focus on the significant roles of these three small RNAs in the regulation of spermatogenesis based on the work of our group and other laboratories. There is increasing evidence indicating that proper small RNA processing is essential for normal spermatogenesis and male fertility. For example, mice lacking Dicer, which is required for both siRNA and miRNA synthesis, have abnormal elongating spermatids and as a

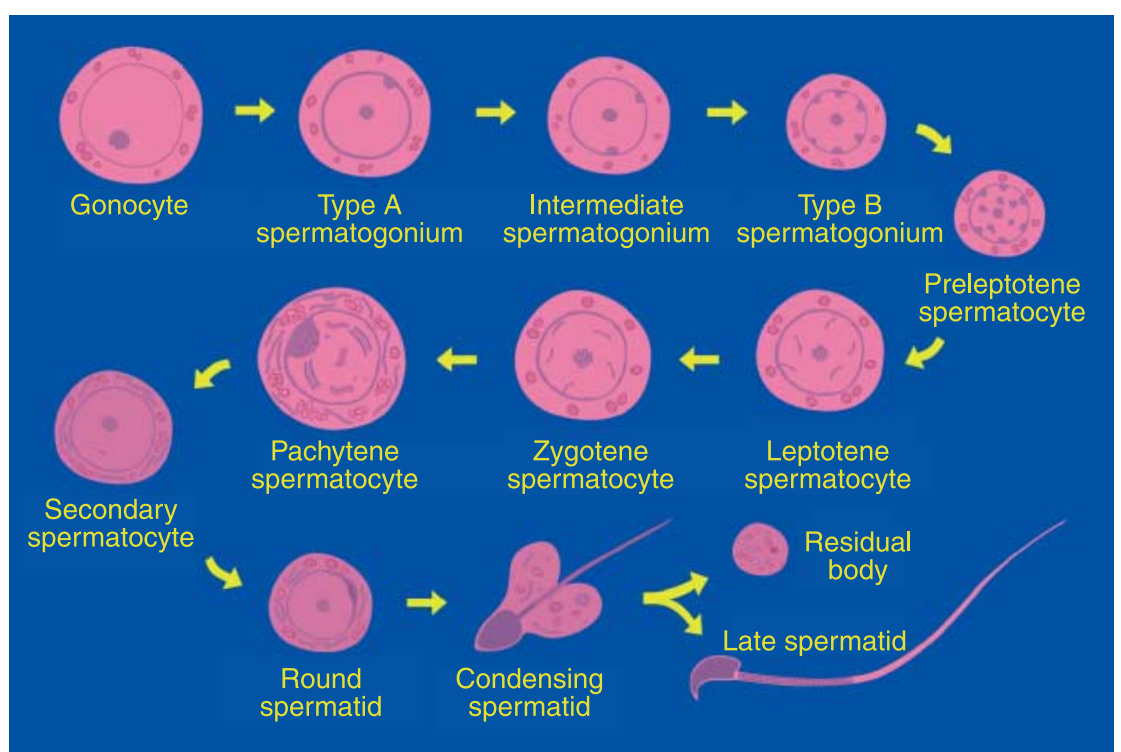

Figure 2 Schematic diagram of rodent spermatogenesis. Spermatogenesis is composed of three phases: the mitotic proliferation of spermatogonia; meiosis with its prolonged meiotic prophase and two reduction divisions (post-meiotic phase) that yield two secondary spermatocytes and then four haploid round spermatids; and spermiogenesis that culminates in the formation of spermatozoa. From Bellvé et al. (1977) with minor modifications. 
Table 1 Comparison of the properties of small interfering RNA (siRNA), microRNA (miRNA), and piwi-interacting RNA (piRNA).

\begin{tabular}{llll}
\hline Properties & siRNA & miRNA & piRNA \\
\hline Length & $\sim 21 \mathrm{nt}$ & $\sim 22 \mathrm{nt}$ & $\sim 24-30 \mathrm{nt}$ \\
Strand & Double-stranded RNA & Single-stranded RNA & Single-stranded RNA \\
Number & NA & Several hundred & $\sim 50000$ \\
Origin & Exogenous RNA & Endogenous RNA & Endogenous RNA \\
Expression & Not expressed in tissues or cells & Various tissues and cells & Spermatocytes, spermatids \\
Binding targets & mRNA & Ago subclass & Piwi subclass \\
Dicer & Dicer required & Dicer required & Dicer independent \\
Function & mRNA degradation or & mRNA instability and & Repression of retrotransposon \& \\
& posttranscriptional silencing & translation inhibition & post-transcriptional regulation \\
\hline
\end{tabular}

The number of siRNAs corresponds to the number of genes, known or unknown, in rodents and humans and each gene can have many siRNAs targeting its various regions. Binding targets refer to the targets that small RNAs bind to, and, as an example, siRNAs can bind to gene transcripts (mRNA). The classification and modes of action of small RNAs are reviewed in Tolia \& Joshua-Tor (2007). NA, not applicable.

consequence acquire male infertility (Maatouk et al. 2008). Moreover, studies on Drosophila Dicer1 mutants indicate that miRNAs are required for division of germline stem cells and for stem cells to bypass the G1/S checkpoint of the cell cycle (Hatfield et al. 2005). Aberrant expression of small RNAs may lead to male infertility and testicular cancer. As a result, the small RNAs identified and yet to be uncovered could become novel attractive targets for male contraception as well as for gene therapy to alleviate male infertility and possibly cure testicular cancer. We present an overview of the synthesis, mechanisms of action, and functions of small RNAs, and then we highlight concerns pertaining to the use of siRNAs in exploring spermatogenesis mechanisms, and finally we review open questions in studies on miRNAs and piRNAs.

\section{Synthesis, mechanisms of action, and functions of miRNA and piRNA}

Unlike siRNAs that are small exogenous dsRNA molecules, miRNAs emerged as a different class of short endogenous single-stranded RNAs. As illustrated in Fig. 3, the biogenesis and action mechanism of miRNAs are a multi-step process: in the nucleus of the cells, the longer RNA molecules are processed by the ribonuclease Drosha to generate $\sim 70$ nt shRNAs (also called precursor miRNAs, i.e. pre-miRNAs); the pre-miRNAs are then transported to the cytoplasm via an exportin5-dependent mechanism (Yi et al. 2003, Lund et al. 2004). In the cytoplasm of the cells, the RNase III enzyme (the Dicer) catalyzes the pre-miRNA to form mature miRNAs (Hutvagner et al. 2001, Carmell \& Hannon 2004, Kim 2005). The resulting mature miRNAs are incorporated

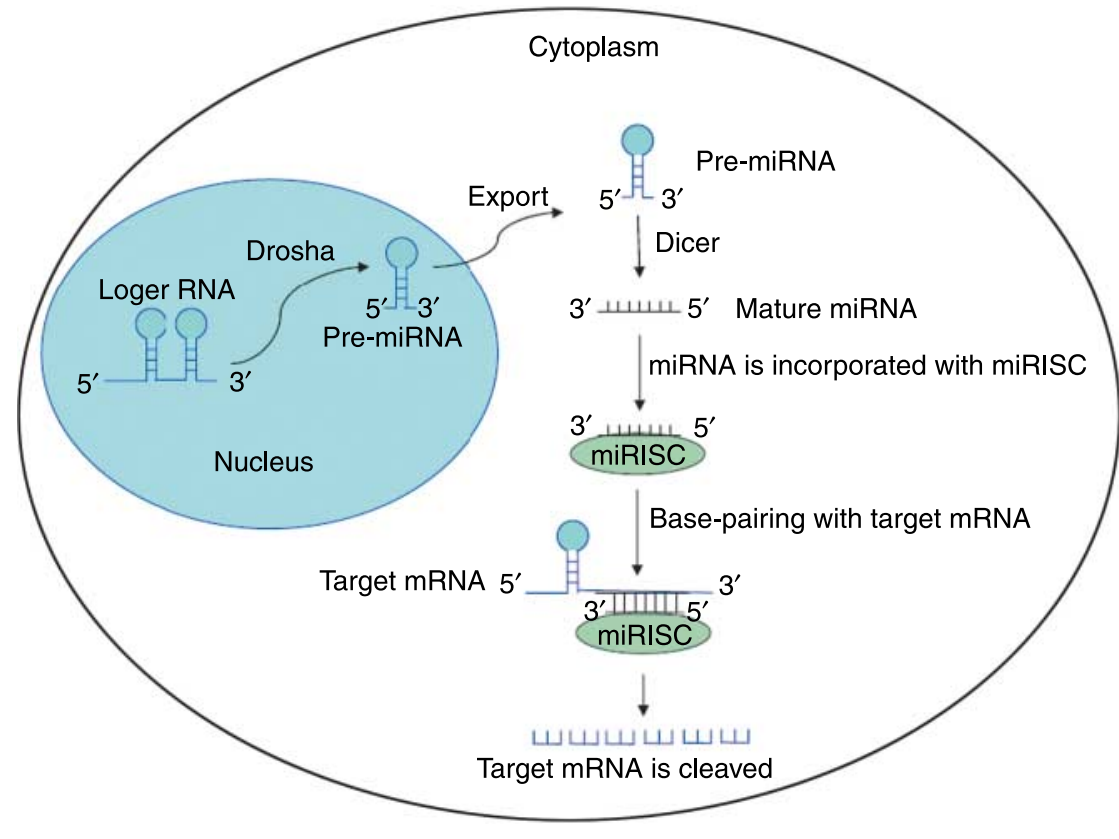

Figure 3 A schematic diagram showing the biogenesis and action mechanism of miRNA in mammalian cells. In the nuclei, pre-miRNA is created by dsRNA-specific ribonuclease Drosha and then exported to the cytoplasm via an exportin-5 dependent mechanism. The premiRNA is catalyzed by Dicer (RNase III enzyme) to form the mature miRNA that is incorporated with the miRNA-induced silencing complex (miRISC). In the resulting complex of miRNA and miRISC, miRNA binds to the target mRNA via base pairing. If miRNA is completely complementary to the seed sequences of mRNA, the target mRNA is cleaved. If the miRNA is not completely complementary to the seed sequences of mRNA, the bound mRNA remains untranslated and results in translation suppression. 
into an effector called miRNA-induced silencing complex (miRISC) composed of Argonaute proteins that have RNase III activity (Martinez et al. 2002, Liu et al. 2004). The Argonaute (Ago) family of proteins consists of two subclasses, the Ago subclass and the Piwi subclass. The Ago subclass interacts with miRNAs and the Piwi subclass interacts with piRNAs. It is believed that miRNAs act as crucial regulators for posttranscriptional gene silencing by base pairing with the 3'-UTRs of target mRNAs to form the RNA duplexes, which leads to endonucleolytic cleavage of the target mRNA (Lai 2002, Carrington \& Ambros 2003). Also, there is another action mechanism by which miRNAs regulate gene expression: some miRNAs are suggested to inhibit mRNA translation directly (Bartel \& Chen 2004), while other miRNAs inhibit the translation initiation through targeting of $\mathrm{m}^{7} \mathrm{G}$-cap recognition (Mathonnet et al. 2007). Importantly, it is now well known that miRNAs have critical functions in many diverse biological processes, including the regulation of stemness ( $\mathrm{Yi}$ et al. 2008), cell proliferation (Brennecke et al. 2003, Lee et al. 2005), differentiation (Chen et al. 2004, Foshay \& Gallicano 2009, Yi et al. 2008), apoptosis (Ambros 2003, Xu et al. 2003), and oncogenesis (Calin et al. 2002, 2004, Esquela-Kerscher \& Slack 2006). Also miRNAs are involved in signaling pathways as illustrated by the Notch and epidermal growth factor (EGF)-signaling pathways regulated by different miRNAs (Lai et al. 2005, Li \& Carthew 2005).

Recent studies have identified another novel class of siRNAs called piRNA, which in males are expressed in mammalian testis during spermatogenesis (Aravin et al. 2006, Girard et al. 2006, Grivna et al. 2006a, Lau et al. 2006). Both piRNAs and miRNAs are endogenous small RNAs, but piRNAs are distinct from miRNAs in their length and expression patterns in that piRNAs are expressed in pachytene spermatocytes and round spermatids (Grivna et al. 2006a), while miRNAs are present in a variety of tissues and a number of cells at various developmental stages.

There are certain common features and some differences among siRNA, miRNAs, and piRNAs. The common features are that these three small RNAs have a strong preference for the $5^{\prime}$ uridine and that they all negatively regulate gene expression, although piRNAs also seem to be able to positively control mRNA stability and translation (Lin 2007). The major differences between the properties of these three small RNAs have been detailed in Table 1.

In Drosophila, the repeat-associated siRNAs (rasiRNAs) have recently been identified (Vagin et al. 2006, Gunawardane et al. 2007). The rasiRNAs can be defined as a piRNA or a subset of piRNAs, since they also bind to the Piwi subfamily proteins and their production is performed in a Dicer-independent manner (Brennecke et al. 2007, Gunawardane et al. 2007, Lin 2007). Although the mechanisms of piRNAs production remain to be elucidated in mammals, a model for the biogenesis of piRNAs has been recently proposed in Drosophila (Brennecke et al. 2007, Gunawardane et al. 2007, Lin 2007). As illustrated in Fig. 4, it is likely that piRNAs are derived from either the repeated DNA sequence elements or complex DNA sequence elements (Brennecke et al. 2007, Gunawardane et al. 2007, Lin 2007). piRNA master control loci of DNA are transcribed and exported from the nucleus to the nuage, an amorphous electron-dense cellular material found in germ cells. The Piwi subfamily Ago protein Argonaute 3 (Ago3) binds to sense-strand transcripts of piRNA to form an Ago3-piRNA complex that guides the slicer-mediated cleavage of target antisense-strand transcripts at an A:U $\mathrm{bp}$, which generates the antisense piRNA precursors, which are a long and single-stranded transcripts with uracyl (U) at the $5^{\prime}$ end (Brennecke et al. 2007,

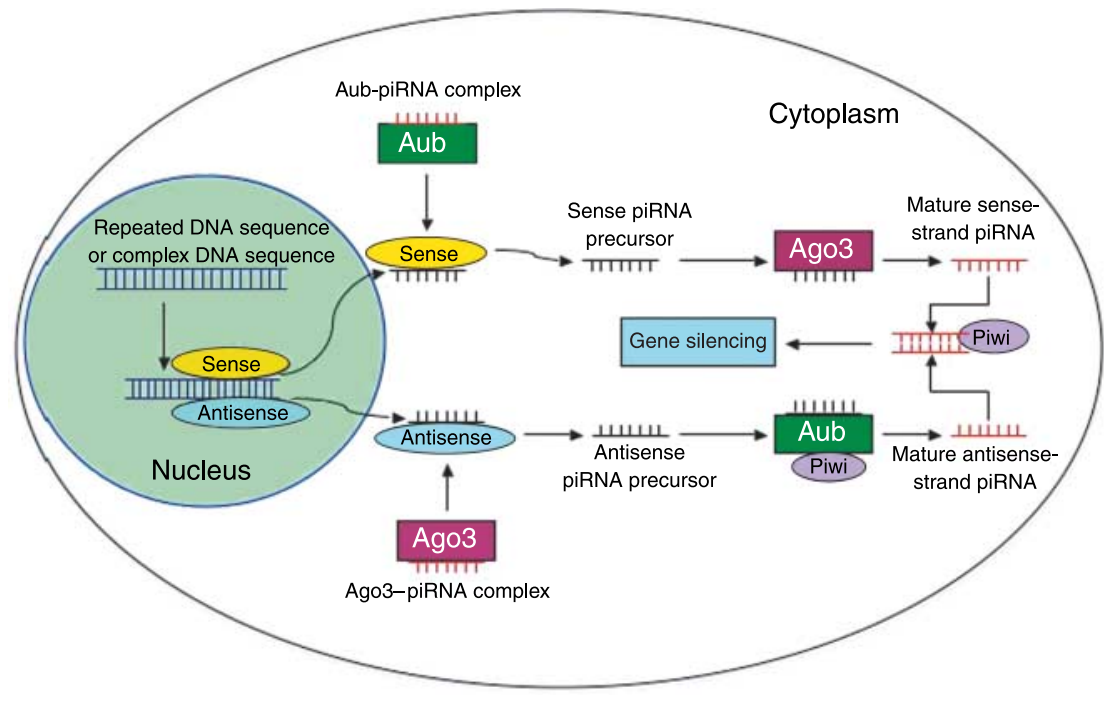

Figure $4 \mathrm{~A}$ schematic diagram showing the biogenesis and action mechanism of piRNA in Drosophila. Argonaute 3 (Ago3) associates with mature sense-strand piRNA to form an Ago3-piRNA complex that cleaves antisense transcripts and produces antisense piRNA precursor. Aubergine (Aub) and piwi associate with antisense piRNA precursor to form a complex that guides the production of mature antisensestrand piRNAs. By contrast, Aub associates with mature antisense-strand piRNA to form an Aub-piRNA complex that cleaves sense transcripts and produces sense piRNA precursor. The sense piRNA precursor associates with Ago3 and forms a complex that guides the production of mature sense-strand piRNAs. piRNA binds to piwi proteins and forms a complex called piwiinteracting RNA complex (piRC) that regulates mRNA expression including gene silencing. 
Gunawardane et al. 2007, Lin 2007). Aubergine and Piwi associate with antisense piRNA precursor to form a complex that is catalyzed by the putative nucleases Squash and Zucchini to produce mature antisense-strand piRNAs (Brennecke et al. 2007, Gunawardane et al. 2007, Lin 2007). By contrast, Aubergine (Aub) binds to antisense-strand transcripts of piRNA to form an Aub-piRNA complex. This complex guides the slicer-mediated cleavage of target sensestrand transcripts, which generates the sense piRNA precursors with an adenine $(\mathrm{A})$ at nt 10 . The sense piRNA precursors associate with Argonaute 3 and are trimmed to mature sense-strand piRNAs (Brennecke et al. 2007, Gunawardane et al. 2007, Lin 2007).

As of yet, the mechanism of piRNA action(s) has not been well documented in mammals. One suggestion is that piRNAs and piwi proteins form a complex called piwi-interacting RNA complex (piRC) that triggers gene silencing (Lau et al. 2006). This hypothesis is supported by the finding that piRC has been extracted and purified from mammalian testis (Lau et al. 2006). piRNAs most likely have diverse functions, including repression of retrotransposons and post-transcriptional regulation including negative and positive regulations (Aravin et al. 2007, Carmell et al. 2007, Lin 2007). However, miRNAs have no effect on the repression of retrotransposons in germ cells (Hayashi et al. 2008), suggesting that miRNAs and their biogenesis have a distinct pathway different from piRNAs in suppressing retrotransposons and germ cell development.

\section{The roles of small RNAs in the regulation of spermatogenesis}

The synthesis and action mechanisms of siRNAs are composed of a series of steps that involve the generation of synthetic siRNAs by in vitro cleavage of long dsRNAs with RNase III endonuclease Dicer or by DNA-based vectors expressing shRNAs that are processed by Dicer into siRNAs (Fig. 5). After transfection of siRNA or shRNA to the cytoplasm, siRNAs are capable of recruiting a multienzyme complex called the RNAinduced silencing complex (RISC) that identifies and cleaves the complementary target mRNA (Plasterk 2002). As a result, the targeted mRNA is degraded and protein synthesis is inhibited (Plasterk 2002), reflecting that siRNAs are critical regulators of post-transcriptional gene silencing. The specific and robust inhibitory effects of RNAi on gene expression make it a valuable research tool in both cell culture and living organisms. In the past few years, the use of siRNA or shRNA has proven to be a powerful tool for knocking down the expression of specific genes in mammalian cells including male germ cells (Elbashir et al. 2002, Plasterk 2002, Oatley et al. 2006, Braydich-Stolle et al. 2007, He et al. 2007, Oatley et al. 2007).

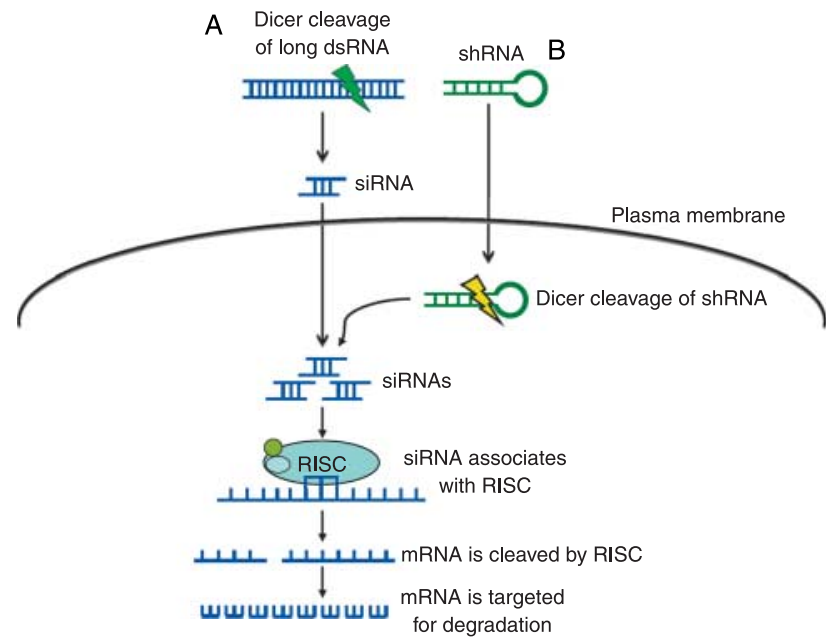

Figure 5 A schematic diagram showing the in vitro synthesis and action mechanism of siRNA and shRNA in mammalian cells. (A) The long dsRNA is cleaved by RNase III endonuclease Dicer to generate synthetic siRNA in vitro. (B) In vitro synthesized shRNA containing 19-29 nt dsRNA stems is processed by Dicer into siRNAs in the cytoplasm of cells. The siRNAs can incorporate into the RNA-induced silencing complex (RISC) that cleaves the targeted mRNA and results in the degradation of cognate mRNA.

Recent advancements using small RNAs have shed light on gene regulation in spermatogenesis. RNAi technology utilizing siRNAs can effectively suppress the expression of specific genes of interest, and the consequences after gene knockdown have been summarized in Table 2. This is exemplified by our recent demonstration that GFRA1 silencing by Gfra 1 siRNAs leads to a switch from renewal to differentiation of mouse SSCs into $A_{1}-A_{4}$ spermatogonia, an initial step of mouse spermatogenesis (He et al. 2007). Nodal knockdown by Nodal siRNAs results in a marked increase in cell apoptosis and a reduction in cell division of mouse SSCs (unpublished observations). Likewise, our group employed siRNAs against Src family kinases and defined the role of the GDNF-signaling pathway in proliferation of mouse SSCs (Braydich-Stolle et al. 2007). In addition, siRNA was utilized to suppress the transcription of $B$ cell CLL/lymphoma 6 member B (Bcl6b), Ets-related molecule (Erm), and LIM homeobox 1 ( $L h x 1$ ) identifying these transcription factors as crucial regulators for SSC self-renewal in vitro (Oatley et al. 2006, 2007).

In somatic Sertoli cells, knockdown of Pard6a or Par3 by their respective siRNAs causes a decrease in expression of the blood-testis barrier (BTB)-associated proteins and demonstrates the role of the Par3/Pard6abased polarity complex in BTB restructuring during spermatogenesis (Wong et al. 2008). Suppression of beta1-integrin by RNAi in Sertoli cells results in occludin redistribution at the Sertoli-Sertoli cell interface and BTB destabilizing (Yan et al. 2008). In an in vivo study, mice treated with siRNA targeted against androgen receptor resulted in reduced expression of FGF2 and 
Table 2 Small interfering RNAs (siRNAs) used to examine functions of specific genes in spermatogenesis.

\begin{tabular}{lll}
\hline siRNAs & Main consequence after gene knockdown & References \\
\hline Androgen receptor & Decrease in FGF2 expression & Gonzalez-Herrera et al. (2006) \\
Bcl6b & Decrease in SSC proliferation & Oatley et al. (2006) \\
Src family kinases & Decrease in SSC proliferation & Braydich-Stolle et al. (2007) \\
Gfra1 & Switch from proliferation to differentiation of & He et al. (2007) \\
Erm & mouse SSCs into A A $_{4}$ spermatogonia & Oatley et al. (2007) \\
Lhx1 & Decrease in SSC maintenance & Oatley et al. (2007) \\
Pard6a or Par3 & Decrease in SSC maintenance & Wong et al. (2008) \\
Beta1-integrin & Decrease in the expression of the blood-testis & barrier (BTB)-associated proteins \\
& Occludin redistribution at the Sertoli cell-Sertoli & Yan et al. (2008)
\end{tabular}

Bcl6b, B cell CLL/lymphoma 6 member B; Gfra1, glial cell line-derived neurotrophic factor family receptor alpha 1; Erm, Ets-related molecule; Lhx 1 , LIM homeobox 1; SSCs, spermatogonial stem cells. In an in vivo study, the FGF2-transgenic mice were injected i.p. daily with siRNAs against androgen receptor to suppress androgen receptor expression. In the in vitro studies, siRNAs against Bcl6b, Src family kinase, Gfra 1, Erm, and Lhx1 were transfected to male germ cells, and siRNAs against Pard6a, Par3, and beta- 1 integrin were transfected to somatic Sertoli cells to silence their expression.

established a role for testosterone in the regulation of FGF2 (Gonzalez-Herrera et al. 2006). Collectively, these studies illustrate that RNAi technology using siRNAs is an effective approach in exploring the function of specific genes in the regulation of spermatogenesis.

Generating expression profiles of miRNAs in male germ cells is a prerequisite for a thorough understanding of their roles in regulating spermatogenesis. Although the functions of miRNAs in the development of male germ cells are still largely unknown, expression profiling studies have identified a number of miRNAs that are enriched in the mammalian testis (Yu et al. 2005, Ro et al. 2007, Yan et al. 2007), including a number of miRNAs that are expressed preferentially in mouse male germ cells, including spermatogonia, pachytene spermatocytes, spermatids, and spermatozoa (Ro et al. 2007), as well as a suite of novel miRNAs in human spermatozoa (Ostermeier et al. 2005). These observations suggest that miRNAs are likely involved in the regulation of gene expression during mitotic, meiotic, and post-meiotic stages of spermatogenesis. The expression profiling of interesting miRNAs in testis has been outlined in Table 3. Specifically, Mir122a is predominately expressed in late-stage male germ cells and it represses the transcription of transition protein 2 (Yu et al. 2005), a marker for round spermatids, suggesting that Mir122a functions in post-meiotic germ cells. Translin (also known as testis-brain RNA-binding protein) has recently been demonstrated to bind to Mir122a and thus increases the in vivo stability of Mir122a (Yu \& Hecht 2008). The expression of Mirn34b is much higher in adult testis compared to the prepuberal testis (Barad et al. 2004), indicating that Mirn34b plays a potential role in the differentiation of male germ cells.

Table 3 An overview of expression profiles of microRNAs (miRNAs) and piwi-interacting RNAs (piRNAs) in testis.

\begin{tabular}{|c|c|c|}
\hline Starting material & Main results & References \\
\hline Prepubertal and adult mouse testis & $\begin{array}{l}\text { Mirn34b expression is much higher in adult mouse } \\
\text { testis than that in prepubertal mouse testis }\end{array}$ & Barad et al. (2004) \\
\hline Human spermatozoa & $\begin{array}{l}68 \text { small RNAs have been identified in } \\
\text { human spermatozoa }\end{array}$ & $\begin{array}{l}\text { Ostermeier et al. (2005) } \\
\text { and Yu et al. (2005) }\end{array}$ \\
\hline Prepubertal and adult mouse testis & $\begin{array}{l}\text { Mir122a is expressed in post-meiotic germ cells } \\
\text { and it reduces transition protein } 2 \text { mRNA }\end{array}$ & Yu et al. (2007) \\
\hline Mouse tissues and germ cells & $\begin{array}{l}22 \text { testis-preferential and } 6 \text { testis-specific miRNAs have } \\
\text { been identified in male germ and Sertoli cells }\end{array}$ & Ro et al. (2007) \\
\hline Immature and adult mouse testis & $\begin{array}{l}14 \text { miRNAs are upregulated and } 5 \text { miRNAs are down- } \\
\text { regulated in immature testis compared to adult testis }\end{array}$ & Yan et al. (2007) \\
\hline Mouse 'spermatogonia' & $\begin{array}{l}\text { miRNA-17-92 cluster and Mirn290-295 cluster are } \\
\text { enriched in spermatogonia of neonatal mice } \\
\text { after } 3 \text { days in culture }\end{array}$ & Hayashi et al. (2008) \\
\hline Mouse spermatogonia, PSc, RSp, and ESp & $\begin{array}{l}\text { piRNAs are expressed in PSc and RSp, but not in } \\
\text { spermatogonia or Esp }\end{array}$ & Aravin et al. (2006) \\
\hline Mouse testis at different developmental stages & $\begin{array}{l}\text { piRNAs are detected at } 14 \mathrm{dpp} \text { and become abundant at } \\
17-18 \text { days, but lost before mature sperm production }\end{array}$ & Girard et al. (2006) \\
\hline Mouse $\mathrm{Mili}^{+/-}$testis at different developmental stages & $\begin{array}{l}\text { piRNAs are only detected in mouse } \mathrm{Mili}^{+/-} \text {testis at } \\
22 \text { or } 24 \mathrm{dpp} \text {, but not at } 16 \mathrm{dpp}\end{array}$ & Grivna et al. (2006b) \\
\hline
\end{tabular}

PSc, pachytene spermatocytes; Rsp, round spermatids; Esp, elongating spermatids; dpp, days post partum. 
Notably, it has recently been demonstrated that miRNA-17-92 cluster and Mirn290-295 cluster are most abundantly expressed in mouse 'spermatogonia' obtained from neonatal mice and then cultured for 3 days, suggesting that these two miRNA clusters play potential roles in regulating proliferation and/or early differentiation of SSCs during spermatogenesis (Hayashi et al. 2008). Furthermore, elongating spermatids exhibit abnormal morphology and motility, and consequently, male infertility occurs in the Dicer1-knockout mice, indicating that both Dicer1 and miRNAs play crucial roles in proper differentiation during spermatogenesis (Maatouk et al. 2008). Using a novel tissue-specific RNAi approach that mimics the principle by which endogenous miRNAs are made, Rao et al. (2006) demonstrated that the Wilms' tumor 1 (WT1) transcription factor plays an essential role in the control of germ cell survival and spermatogenesis. WT1 knockdown mice suffered from increased germ cell apoptosis, a loss of the adherens junction complex between germ cells and Sertoli cells, and impaired fertility. Together, these studies further indicate that normal miRNA biogenesis is required for proper spermatogenesis.

The roles of piRNAs in spermatogenesis are supported by the known functions of their partner, the Piwi proteins. Accumulating evidence indicate that the piwi subfamily proteins, including MIWI, MIWI2, and MILI, are required for stem cell self-renewal and the development of male germ cells in invertebrates (Cox et al. 1998, Reddien et al. 2005, Aravin et al. 2006, Klattenhoff \& Theurkauf 2008). In mammals, MIWI, MIWI2, and MILI proteins are expressed in mid- and late-stage germ cells and are essential for mouse spermatogenesis (Deng \& Lin 2002, Kuramochi-Miyagawa et al. 2004, Carmell et al. 2007). The MIWI protein is present in the cytoplasm of spermatocytes as well as in the chromatoid body and cytoplasm of round spermatids, and more importantly, MIWI associates with piRNAs in the translation machinery and mRNA stability (Grivna et al. 2006b). Notably, spermatogenesis is arrested at the pachytene spermatocyte stage in Mili-knockout mice (KuramochiMiyagawa et al. 2004) and at the round spermatid stage without elongated spermatids or mature spermatozoa production in Miwi-deficient mice (Deng \& Lin 2002), while Miwi2-deficient mice display a defect in the early prophase of meiosis I and a marked and progressive loss of germ cells with age (Carmell et al. 2007). Thus, it is likely that piRNAs, the partners of piwi subfamily proteins, are potentially involved in regulating the processes of meiosis and post-meiosis of male germ cell development. This view is supported by the fact that piRNAs are expressed in male germ cells, particularly pachytene spermatocytes and round spermatids as summarized in Table 3 (Aravin et al. 2006, Girard et al. 2006, Grivna et al. 2006a), and they play a role in repression of retrotransposons during spermatogenesis (Aravin et al. 2007, Carmell et al. 2007). In addition, the small non-coding RNAs Nct1 and Nct2 have recently been suggested to be piRNA precursors and they are expressed predominantly in pachytene spermatocytes (Iguchi et al. 2007, Xu et al. 2008), which also suggests a role of piRNAs in the regulation of the meiotic stage in spermatogenesis. However, Nct1/2-deficient mice display a decrease in a small cluster of piRNAs (e.g. antisense LINE 1-related repeat sequence piRNA) in chromosome 2, but does not affect mouse spermatogenesis or fertility, suggesting that these piRNAs on chromosome 2 are necessary to maintain transposon silencing (Xu et al. 2008).

In addition, another novel class of small RNAs has recently been identified after sequencing 1111 clones of small RNAs from mouse testis in that their sequences and genome mapping data do not match those of any previously described small RNAs (Watanabe et al. 2006). Since these small RNAs are expressed only in mouse male germ cells, this class of small RNAs is named germline small RNAs (gsRNAs; Watanabe et al. 2006). gsRNAs have distinct features compared to other small RNAs: i) the mean length of gsRNAs is longer than miRNAs and siRNAs; ii) gsRNAs have a strand bias unlinked to a stem-loop structure. gsRNAs are restricted to the period from pachytene spermatocytes to round spermatids (Watanabe et al. 2006). This specific expression pattern would suggest specific roles of gsRNAs in the meiotic and post-meiotic phases of spermatogenesis. However, the biogenesis, mechanisms, and the functions of gsRNAs are still unclear.

\section{Potential applications of small RNAs to male contraception and treatment for male infertility and testicular cancer}

There are three main applications for RNAi using siRNAs: i) basic research - to further examine the potential roles of specific genes and signaling pathways in the regulation of spermatogenesis; ii) male contraception - RNAi can be used as an effective tool to develop new approaches for male contraception. Recently, RNAi in vivo has been demonstrated to work effectively in male germ cells during mitosis and meiosis as well as in haploid cells (Shoji et al. 2005). Thus, male contraception may be achieved by injecting directly into the testis synthetic siRNAs or shRNAs to knock down the expression of genes that are required for normal spermatogenesis. iii) Gene therapy for male infertility by RNAi use in vivo - it is necessary to diminish expression of diseased genes. Notably, a number of genes, e.g. clusterin, Aard, Akr1b3, Defb19, Itga6, Itgb1, Maged2, Rbm3, and Vim, have been shown to be upregulated in mouse models of male infertility as we summarized in a previous review (He et al. 2006). Therefore, it is possible to suppress expression of 
diseased gene transcripts among the upregulated genes in vivo by injecting siRNAs or using a DNA vector expressing shRNAs and determine whether the sterility of mice can be rescued after RNAi.

Given that miRNAs and piRNAs play potentially important roles in spermatogenesis, inhibitors that antagonize these small RNAs also may be used as future male contraceptives. One important advantage of using inhibitors for miRNAs and piRNAs as an approach for male contraception is that there would most likely be fewer side effects, since a number of miRNAs and piRNAs are expressed exclusively in testis but not in other tissues, and more importantly, piRNAs are expressed only in male mid- and late-stage germ cells (Aravin et al. 2006, Girard et al. 2006, Grivna et al. 2006a). It has been demonstrated that potent miRNAs inhibitors, the complementary oligos with miRNAs such as antagomir, can effectively suppress the expression of targeted miRNAs via specific binding (Krutzfeldt et al. $2005,2007)$. In addition, interference of the biogenesis of miRNAs may be used as an approach for male contraception as evidence by the fact that impaired miRNA processing (e.g. the depletion of Dicer1) results in mouse male infertility (Maatouk et al. 2008, Otsuka et al. 2008). Since there is high conservation in miRNAs between human and mice, the knowledge derived from mouse miRNAs in male infertility should be applicable to humans.

miRNAs may also play roles in carcinogenesis of human testicular cancer. Especially, noted are Mirn322 and Mirn323 that are suggested to be potential novel oncogenes participating in the development of human testicular germ cell tumors (GCTs; Voorhoeve et al. 2006, 2007). In a genetic screen for miRNAs that cooperate with oncogenes in cellular transformation, MIRNA372 and MIRNA373 were demonstrated to be possible oncogenes that are involved in the development of human testicular GCTs (Voorhoeve et al. 2006). Differential expression patterns of 156 miRNAs in a series of type II and III testicular GCTs have been uncovered using a quantitative PCR-based approach (Gillis et al. 2007), and it is highly informative for distinguishing type II and III GCTs. Thus, to uncover the differential expression profiling of all miRNAs between human testicular cancer and normal men may have prognostic value and may provide novel molecular signatures for the diagnosis, prevention, and eventually gene therapy of human testicular cancer. In addition, the dead-end (Dnd1) gene was shown to be essential for maintaining the viability of male germ cells, and Dnd1deficient mice exhibit loss of germ cells and testicular GCTs (Youngren et al. 2005). The Dnd1-encoded protein DND1 interacts with APOBEC3 and binds to mRNA to inhibit miRNA-mediated repression of mRNA, which is suggested to be one mechanism for preventing GCT development (Bhattacharya et al. 2008).

\section{Concerns pertaining to the use of siRNA and open questions for miRNAs and piRNAs research on spermatogenesis mechanisms}

A key to a successful RNAi study is closely related to a high transfection efficiency of the siRNAs, and this depends on the cell type and the transfection reagents used. Lipofectamine 2000, which was originally developed for the delivery of plasmid DNA, has been shown to work effectively with siRNAs (Bonetta 2005). In an in vitro study, Brinster et al. used Lipofectamine 2000 to transfect siRNAs against Bcl6b, Erm, and Lhx1 to mouse SSCs, and they got $52-87 \%$ of decrease in these gene transcripts as measured by quantitative RT-PCR (Oatley et al. 2007). We showed that siRNAs against Gfra1 could be efficiently transfected into type A spermatogonia using Lipofectamine 2000 , as shown by a high uptake of the BLOCK-iT fluorescent oligo, whose fluorescent signal correlates with the delivery of active siRNAs (He et al. 2007). In one in vivo study, DNA vectors expressing shRNAs were introduced into mouse testes via DNA injection and electroporation, and more than $80 \%$ of mRNA reduction in the reporter gene EGFP and DsRed2 was observed in spermatogonia, spermatocytes, and round spermatids (Shoji et al. 2005), suggesting that the electroporation approach is effective for silencing gene expression of spermatogenic cells in vivo. In another in vivo study, the FGF2-transgenic mice were injected intraperitoneously daily with $100 \mu \mathrm{l}$ of solution of siRNAs against androgen receptor in isotonic saline solution, and $70 \%$ efficiency of androgen receptor protein depletion was obtained as assayed by western blots (Gonzalez-Herrera et al. 2006), which illustrates that RNAi is feasible for suppressing gene expression of somatic cells in vivo. The major obstacle to achieving RNAi with longer dsRNAs in mammalian cells is that they cause non-specific mRNA degradation and a general shutdown of host cell protein synthesis. One of the solutions to this issue is to use siRNA with $<23 \mathrm{nt}$ to trigger specific gene silencing without causing an interferon response (Elbashir et al. 2002, Heidel et al. 2004). Sequence specific off-target effect is another concern for siRNA research. The confidence of the RNAi data can be obtained by individually transfecting two or more siRNAs targeting different sequences of the gene of interest. Also, proper controls are needed for every RNAi study, e.g. a negative control siRNA that is neither homologous to anything in the vertebrate transcriptome nor induces a stress response would be necessary for the control of non-specific effects of gene expression caused by siRNA delivery. Likewise, positive control siRNAs are needed to ensure that siRNAs are transfected efficiently and that the gene silencing system is working.

There are many interesting and important aspects to pursue in miRNA research on spermatogenesis mechanisms. The future directions of particular 
importance may include: i) to establish cell- and stagespecific expression profiling of miRNAs during mammalian spermatogenesis using miRNA microarray or real-time RT-PCR; for a small amount of starting material, it is possible to analyze the miRNA expression pattern in a single cell such as a stem cell by using a realtime PCR-based 220-plex miRNA expression profiling method (Chen et al. 2005, Tang et al. 2006a, 2006b, Hayashi et al. 2008); ii) to explore the potential roles of miRNAs in regulating renewal versus differentiation of male germline stem cells during spermatogenesis; iii) to identify the regulatory gene targets by miRNAs in male germ cells and somatic cells; and iv) to unveil the potential roles of miRNAs in the testis carcinogenesis. Likewise, there are a number of questions that warrant further investigation on piRNAs. First, the action mechanisms by which piRNA silences gene expression need to be elucidated. Second, it is imperative to uncover the cell-specific expression profiling of piRNAs in male germ cells during mammalian spermatogenesis. In zebrafish ovaries, piRNAs are required for stem cell renewal during oogenesis (Klattenhoff \& Theurkauf 2008). A possible role for piRNA in mammalian SSC renewal remains unknown. Finally, and more importantly, to explore the potential roles of piRNAs in the meiotic and post-meiotic steps of germ-cell development and in the oncogenesis of GCTs. Overall, such studies mentioned above would provide novel insights into molecular mechanisms governing mammalian spermatogenesis and attractive targets for male contraception and eventually render gene therapy of male infertility and testicular cancer.

\section{Conclusion}

Recent advancements on small RNAs have revealed the biological significance of these molecules in the regulation of spermatogenesis. The use of siRNA technology for gene silencing and the understanding of the biogenesis, mechanisms of action, expression profiles, and functions of miRNAs and piRNAs have provided us with more and more insights into spermatogenesis regulated by small RNAs. The advancements made in the small RNAs-regulating spermatogenesis would be helpful for a better understanding of the etiology of male infertility and testicular cancer. To further explore the functions and the potential targets of small RNAs identified and yet to be unveiled and to establish a list of cell- and stage-specific distribution of these small RNAs in the testis would provide more insights into the molecular regulation of spermatogenesis, and may eventually lead to the development of novel male contraception and gene therapy for male infertility and testicular cancer.

\section{Declaration of interest}

The authors declare that there is no conflict of interest that would prejudice the impartiality of this scientific work.

\section{Funding}

This work was supported by NIH Grant HD 33728 to Dr Dym.

\section{References}

Ambros V 2003 MicroRNA pathways in flies and worms: growth, death, fat, stress, and timing. Cell 113 673-676.

Aravin A, Gaidatzis D, Pfeffer S, Lagos-Quintana M, Landgraf P, lovino N, Morris P, Brownstein MJ, Kuramochi-Miyagawa S, Nakano T et al. 2006 A novel class of small RNAs bind to MILI protein in mouse testes. Nature 442 203-207.

Aravin AA, Sachidanandam R, Girard A, Fejes-Toth K \& Hannon GJ 2007 Developmentally regulated piRNA clusters implicate MILI in transposon control. Science 316 744-747.

Barad O, Meiri E, Avniel A, Aharonov R, Barzilai A, Bentwich I, Einav U, Gilad S, Hurban P, Karov Y et al. 2004 MicroRNA expression detected by oligonucleotide microarrays: system establishment and expression profiling in human tissues. Genome Research 14 2486-2494.

Bartel DP \& Chen CZ 2004 Micromanagers of gene expression: the potentially widespread influence of metazoan microRNAs. Nature Reviews. Genetics 5 396-400.

Bellvé AR, Cavicchia JC, Millette CF, O'Brien DA, Bhatnagar YM \& Dym M 1977 Spermatogenic cells of the prepuberal mouse. Isolation and morphological characterization. Journal of Cell Biology 74 68-85.

Betel D, Sheridan R, Marks DS \& Sander C 2007 Computational analysis of mouse piRNA sequence and biogenesis. PLoS Computational Biology 3 e222.

Bhattacharya C, Aggarwal S, Kumar M, Ali A \& Matin A 2008 Mouse apolipoprotein $\mathrm{B}$ editing complex 3 (APOBEC3) is expressed in germ cells and interacts with dead-end (DND1). PLOS ONE 3 e2315.

Bonetta L 2005 The inside scoop-evaluating gene delivery methods. Nature Methods 2 875-883.

Braydich-Stolle L, Kostereva N, Dym M \& Hofmann MC 2007 Role of Src family kinases and $\mathrm{N}-\mathrm{Myc}$ in spermatogonial stem cell proliferation. Developmental Biology 304 34-45.

Brennecke J, Hipfner DR, Stark A, Russell RB \& Cohen SM 2003 Bantam encodes a developmentally regulated microRNA that controls cell proliferation and regulates the proapoptotic gene hid in Drosophila. Cell 113 25-36.

Brennecke J, Aravin AA, Stark A, Dus M, Kellis M, Sachidanandam R \& Hannon GJ 2007 Discrete small RNA-generating loci as master regulators of transposon activity in Drosophila. Cell 128 1089-1103.

Calin GA, Dumitru CD, Shimizu M, Bichi R, Zupo S, Noch E, Aldler H, Rattan S, Keating M, Rai K et al. 2002 Frequent deletions and downregulation of micro- RNA genes miR15 and miR16 at 13q14 in chronic lymphocytic leukemia. PNAS 99 15524-15529.

Calin GA, Liu CG, Sevignani C, Ferracin M, Felli N, Dumitru CD, Shimizu M, Cimmino A, Zupo S, Dono M et al. 2004 MicroRNA profiling reveals distinct signatures in B cell chronic lymphocytic leukemias. PNAS 101 11755-11760.

Carmell MA \& Hannon GJ 2004 RNase III enzymes and the initiation of gene silencing. Nature Structural and Molecular Biology 11 214-218.

Carmell MA, Girard A, van de Kant HJ, Bourc'his D, Bestor TH, de Rooij DG \& Hannon GJ 2007 MIWI2 is essential for spermatogenesis and repression of transposons in the mouse male germline. Developmental Cell 12 503-514.

Carrington JC \& Ambros V 2003 Role of microRNAs in plant and animal development. Science 301 336-338.

Chen CZ, Li L, Lodish HF \& Bartel DP 2004 MicroRNAs modulate hematopoietic lineage differentiation. Science 303 83-86. 
Chen C, Ridzon DA, Broomer AJ, Zhou Z, Lee DH, Nguyen JT, Barbisin M, Xu NL, Mahuvakar VR, Andersen MR et al. 2005 Real-time quantification of microRNAs by stem-loop RT-PCR. Nucleic Acids Research 33 e179.

Cox DN, Chao A, Baker J, Chang L, Qiao D \& Lin H 1998 A novel class of evolutionarily conserved genes defined by piwi are essential for stem cell self-renewal. Genes and Development 12 3715-3727.

Deng W \& Lin H 2002 Miwi, a murine homolog of piwi, encodes a cytoplasmic protein essential for spermatogenesis. Developmental Cell 2 819-830.

Elbashir SM, Harborth J, Weber K \& Tuschl T 2002 Analysis of gene function in somatic mammalian cells using small interfering RNAs. Methods 26 199-213.

Esquela-Kerscher A \& Slack FJ 2006 Oncomirs - microRNAs with a role in cancer. Nature Reviews. Cancer 6 259-269.

Foshay K \& Gallicano GI 2009 miR-17 Family miRNAs are expressed during early mammalian development and regulate stem cell differentiation. Developmental Biology 18 201-204.

Gillis AJ, Stoop HJ, Hersmus R, Oosterhuis JW, Sun Y, Chen C, Guenther S, Sherlock J, Veltman I, Baeten J et al. 2007 High-throughput microRNAome analysis in human germ cell tumours. Journal of Pathology 213 319-328.

Girard A, Sachidanandam R, Hannon GJ \& Carmell MA 2006 A germlinespecific class of small RNAs binds mammalian Piwi proteins. Nature $\mathbf{4 4 2}$ 199-202.

Gonzalez-Herrera IG, Prado-Lourenco L, Pileur F, Conte C, Morin A, Cabon F, Prats H, Vagner S, Bayard F, Audigier S et al. 2006 Testosterone regulates FGF-2 expression during testis maturation by an IRESdependent translational mechanism. FASEB Journal 20 476-478.

Grivna ST, Beyret E, Wang Z \& Lin H 2006a A novel class of small RNAs in mouse spermatogenic cells. Genes and Development 20 1709-1714.

Grivna ST, Pyhtila B \& Lin H $2006 b$ MIWI associates with translational machinery and PIWI-interacting RNAs (piRNAs) in regulating spermatogenesis. PNAS 103 13415-13420.

Gunawardane LS, Saito K, Nishida KM, Miyoshi K, Kawamura Y, Nagami T, Siomi H \& Siomi MC 2007 A slicer-mediated mechanism for repeatassociated siRNA $5^{\prime}$ end formation in Drosophila. Science 315 1587-1590.

Hatfield SD, Shcherbata HR, Fischer KA, Nakahara K, Carthew RW \& Ruohola-Baker H 2005 Stem cell division is regulated by the microRNA pathway. Nature 435 974-978.

Hayashi K, Chuva de Sousa Lopes SM, Kaneda M, Tang F, Hajkova P, Lao K, O'Carroll D, Das PP, Tarakhovsky A, Miska EA et al. 2008 MicroRNA biogenesis is required for mouse primordial germ cell development and spermatogenesis. PLOS ONE 3 e1738.

He Z, Chan WY \& Dym M 2006 Microarray technology offers a novel tool for the diagnosis and identification of therapeutic targets for male infertility. Reproduction 132 11-19.

He Z, Jiang J, Hofmann MC \& Dym M 2007 Gfra1 silencing in mouse spermatogonial stem cells results in their differentiation via the inactivation of RET tyrosine kinase. Biology of Reproduction 77 723-733.

Heidel JD, Hu S, Liu XF, Triche TJ \& Davis ME 2004 Lack of interferon response in animals to naked siRNAs. Nature Biotechnology 22 1579-1582.

Huckins C 1971 The spermatogonial stem cell population in adult rats. I. Their morphology, proliferation and maturation. Anatomical Record 169 533-557.

Hutvagner G, McLachlan J, Pasquinelli AE, Balint E, Tuschl T \& Zamore PD 2001 A cellular function for the RNA-interference enzyme Dicer in the maturation of the let-7 small temporal RNA. Science 293 834-838.

Iguchi N, Xu M, Hori T \& Hecht NB 2007 Noncoding RNAs of the mammalian testis: the meiotic transcripts Nct1 and Nct2 encode piRNAs. Annals of the New York Academy of Sciences 1120 84-94.

Kim VN 2005 MicroRNA biogenesis: coordinated cropping and dicing. Nature Reviews. Molecular Cell Biology 6 376-385.

Klattenhoff C \& Theurkauf W 2008 Biogenesis and germline functions of piRNAs. Development 135 3-9.

Krutzfeldt J, Rajewsky N, Braich R, Rajeev KG, Tuschl T, Manoharan M \& Stoffel M 2005 Silencing of microRNAs in vivo with 'antagomirs'. Nature 438 685-689.
Krutzfeldt J, Kuwajima S, Braich R, Rajeev KG, Pena J, Tuschl T, Manoharan M \& Stoffel M 2007 Specificity, duplex degradation and subcellular localization of antagomirs. Nucleic Acids Research 35 2885-2892.

Kuramochi-Miyagawa S, Kimura T, Ijiri TW, Isobe T, Asada N, Fujita Y, Ikawa M, Iwai N, Okabe M, Deng W et al. 2004 Mili, a mammalian member of piwi family gene, is essential for spermatogenesis. Development 131 839-849.

Lagos-Quintana M, Rauhut R, Lendeckel W \& Tuschl T 2001 Identification of novel genes coding for small expressed RNAs. Science 294 853-858.

Lai EC 2002 Micro RNAs are complementary to $3^{\prime}$ UTR sequence motifs that mediate negative post-transcriptional regulation. Nature Genetics $\mathbf{3 0}$ 363-364.

Lai EC, Tam B \& Rubin GM 2005 Pervasive regulation of Drosophila notch target genes by GY-box-Brd-box-, and K-box-class microRNAs. Genes and Development 19 1067-1080.

Lau NC, Seto AG, Kim J, Kuramochi-Miyagawa S, Nakano T, Bartel DP \& Kingston RE 2006 Characterization of the piRNA complex from rat testes. Science 313 363-367.

Lee RC, Feinbaum RL \& Ambros V 1993 The C. elegans heterochronic gene lin-4 encodes small RNAs with antisense complementarity to lin-14. Cell 75 843-854.

Lee YS, Kim HK, Chung S, Kim KS \& Dutta A 2005 Depletion of human micro-RNA miR-125b reveals that it is critical for the proliferation of differentiated cells but not for the down-regulation of putative targets during differentiation. Journal of Biological Chemistry 280 16635-16641.

Lewis BP, Burge CB \& Bartel DP 2005 Conserved seed pairing, often flanked by adenosines, indicates that thousands of human genes are microRNA targets. Cell 120 15-20.

Li X \& Carthew RW 2005 A microRNA mediates EGF receptor signaling and promotes photoreceptor differentiation in the Drosophila eye. Cell 123 1267-1277.

Lin H 2007 piRNAs in the germ line. Science 316397.

Liu J, Carmell MA, Rivas FV, Marsden CG, Thomson JM, Song JJ, Hammond SM, Joshua-Tor L \& Hannon GJ 2004 Argonaute2 is the catalytic engine of mammalian RNAi. Science 305 1437-1441.

Lund E, Guttinger S, Calado A, Dahlberg JE \& Kutay U 2004 Nuclear export of microRNA precursors. Science 303 95-98.

Maatouk DM, Loveland KL, McManus MT, Moore K \& Harfe BD 2008 Dicer1 is required for differentiation of the mouse male germline. Biology of Reproduction 79 696-703.

Martinez J, Patkaniowska A, Urlaub H, Luhrmann R \& Tuschl T 2002 Single-stranded antisense siRNAs guide target RNA cleavage in RNAi. Cell 110 563-574.

Mathonnet G, Fabian MR, Svitkin YV, Parsyan A, Huck L, Murata T, Biffo S, Merrick WC, Darzynkiewicz E, Pillai RS et al. 2007 MicroRNA inhibition of translation initiation in vitro by targeting the cap-binding complex elF4F. Science 317 1764-1767.

Oakberg EF 1971 Spermatogonial stem-cell renewal in the mouse. Anatomical Record 169 515-531.

Oatley JM, Avarbock MR, Telaranta Al, Fearon DT \& Brinster RL 2006 Identifying genes important for spermatogonial stem cell self-renewal and survival. PNAS 103 9524-9529.

Oatley JM, Avarbock MR \& Brinster RL 2007 Glial cell line-derived neurotrophic factor regulation of genes essential for self-renewal of mouse spermatogonial stem cells is dependent on Src family kinase signaling. Journal of Biological Chemistry 282 25842-25851.

Ostermeier GC, Goodrich RJ, Moldenhauer JS, Diamond MP \& Krawetz SA 2005 A suite of novel human spermatozoal RNAs. Journal of Andrology 26 70-74.

Otsuka M, Zheng M, Hayashi M, Lee JD, Yoshino O, Lin S \& Han J 2008 Impaired microRNA processing causes corpus luteum insufficiency and infertility in mice. Journal of Clinical Investigation 118 1944-1954.

Plasterk RH 2002 RNA silencing: the genome's immune system. Science 296 1263-1265.

Plasterk RH 2006 Micro RNAs in animal development. Cell 124 877-881.

Rao MK, Pham J, Imam JS, MacLean JA, Murali D, Furuta Y, Sinha-Hikim AP \& Wilkinson MF 2006 Tissue-specific RNAi reveals that WT1 expression in nurse cells controls germ cell survival and spermatogenesis. Genes and Development 20 147-152. 
Reddien PW, Oviedo NJ, Jennings JR, Jenkin JC \& Sanchez Alvarado A 2005 SMEDWI-2 is a PIWI-like protein that regulates planarian stem cells. Science 310 1327-1330.

Ro S, Park C, Sanders KM, McCarrey JR \& Yan W 2007 Cloning and expression profiling of testis-expressed microRNAs. Developmental Biology 311 592-602.

de Rooij DG \& Grootegoed JA 1998 Spermatogonial stem cells. Current Opinion in Cell Biology 10 694-701.

Shoji M, Chuma S, Yoshida K, Morita T \& Nakatsuji N 2005 RNA interference during spermatogenesis in mice. Developmental Biology 282 524-534.

Tang F, Hajkova P, Barton SC, Lao K \& Surani MA 2006a MicroRNA expression profiling of single whole embryonic stem cells. Nucleic Acids Research 34 e9.

Tang F, Hajkova P, Barton SC, O'Carroll D, Lee C, Lao K \& Surani MA $2006 b$ 220-plex microRNA expression profile of a single cell. Nature Protocols 1 1154-1159.

Tolia NH \& Joshua-Tor L 2007 Slicer and the argonautes. Nature Chemical Biology 3 36-43.

Vagin VV, Sigova A, Li C, Seitz H, Gvozdev V \& Zamore PD 2006 A distinct small RNA pathway silences selfish genetic elements in the germline. Science 313 320-324.

Voorhoeve PM, le Sage C, Schrier M, Gillis AJ, Stoop H, Nagel R, Liu YP, van Duijse J, Drost J, Griekspoor A et al. 2006 A genetic screen implicates miRNA-372 and miRNA-373 as oncogenes in testicular germ cell tumors. Cell 124 1169-1181.

Voorhoeve PM, le Sage C, Schrier M, Gillis AJ, Stoop H, Nagel R, Liu YP, van Duijse J, Drost J, Griekspoor A et al. 2007 A genetic screen implicates miRNA-372 and miRNA-373 as oncogenes in testicular germ cell tumors. Advances in Experimental Medicine and Biology 604 17-46.

Watanabe T, Takeda A, Tsukiyama T, Mise K, Okuno T, Sasaki H, Minami N \& Imai H 2006 Identification and characterization of two novel classes of small RNAs in the mouse germline: retrotransposon-derived siRNAs in oocytes and germline small RNAs in testes. Genes and Development 20 1732-1743.

Wightman B, Ha I \& Ruvkun G 1993 Posttranscriptional regulation of the heterochronic gene lin-14 by lin-4 mediates temporal pattern formation in C. elegans. Cell 75 855-862.

Wong EW, Mruk DD, Lee WM \& Cheng CY 2008 Par3/Pard6a polarity complex coordinates apical ectoplasmic specialization and blood-testis barrier restructuring during spermatogenesis. PNAS 105 9657-9662.
Xu P, Vernooy SY, Guo M \& Hay BA 2003 The Drosophila microRNA Mir-14 suppresses cell death and is required for normal fat metabolism. Current Biology 13 790-795.

Xu M, You Y, Hunsicker P, Hori T, Small C, Griswold MD \& Hecht NB 2008 Mice deficient for a small cluster of Piwi-interacting RNAs implicate Piwi-interacting RNAs in transposon control. Biology of Reproduction 79 $51-57$.

Yan N, Lu Y, Sun H, Tao D, Zhang S, Liu W \& Ma Y 2007 A microarray for microRNA profiling in mouse testis tissues. Reproduction $\mathbf{1 3 4}$ 73-79.

Yan HH, Mruk DD, Wong EW, Lee WM \& Cheng CY 2008 An autocrine axis in the testis that coordinates spermiation and blood-testis barrier restructuring during spermatogenesis. PNAS 105 8950-8955.

Yi R, Qin Y, Macara IG \& Cullen BR 2003 Exportin-5 mediates the nuclear export of pre-microRNAs and short hairpin RNAs. Genes and Development 17 3011-3016.

Yi R, Poy MN, Stoffel M \& Fuchs E 2008 A skin microRNA promotes differentiation by repressing 'stemness'. Nature 452 225-229.

Youngren KK, Coveney D, Peng X, Bhattacharya C, Schmidt LS, Nickerson ML, Lamb BT, Deng JM, Behringer RR, Capel B et al. 2005 The Ter mutation in the dead end gene causes germ cell loss and testicular germ cell tumours. Nature 435 360-364.

Yu Z \& Hecht NB 2008 The DNA/RNA-binding protein, translin, binds microRNA122a and increases its in vivo stability. Journal of Andrology $29572-579$.

Yu Z, Raabe T \& Hecht NB 2005 MicroRNA Mirn122a reduces expression of the posttranscriptionally regulated germ cell transition protein 2 (Tnp2) messenger RNA (mRNA) by mRNA cleavage. Biology of Reproduction 73 427-433.

Yu J, Vodyanik MA, Smuga-Otto K, Antosiewicz-Bourget J, Frane JL, Tian S, Nie J, Jonsdottir GA, Ruotti V, Stewart R et al. 2007 Induced pluripotent stem cell lines derived from human somatic cells. Science $\mathbf{3 1 8}$ 1917-1920.

Received 26 November 2008

First decision 16 January 2009

Revised manuscript received 20 February 2009

Accepted 24 March 2009 